\title{
A Pseudo-TDMA MAC Protocol Using Randomly Determined Transmission Times for Landslide Prediction Wireless Sensor Networks
}

\author{
David ASANO $^{\dagger \text { a) }}$, Member, Daichi KUROYANAGI ${ }^{\dagger \dagger}$, Nonmember, Hikofumi SUZUKI ${ }^{\dagger \dagger \dagger}$, Member $^{\dagger}$, \\ Eiki MOTOYAMA ${ }^{\dagger \dagger}$, Nonmember, and Yasushi FUWA ${ }^{\dagger \dagger}$, Member
}

\begin{abstract}
SUMMARY Landslides during heavy rainfall cause a great amount of damage in terms of both property and human life. To predict landslide disasters, we designed and implemented a wireless sensor network using our existing highly fault tolerant ad-hoc network. Since many sensors must be used, we propose a new MAC protocol that allows the network to support more sensor terminals. Our protocol is a hybrid CSMA/Psuedo-TDMA scheme which allows the terminals to decide their transmission timing independently in a random fashion. A timing beacon is not required, so power consumption can be reduced. Simulation results show that the number of terminals supported by the network can be greatly increased.

key words: MAC protocol, TDMA, CSMA, wireless sensor networks, landslides, disaster prediction
\end{abstract}

\section{Introduction}

In Japan, many natural disasters such as landslides and earthquakes occur every year. Even though the central and regional governments have introduced some disaster prediction and prevention facilities, the resulting cost in human life and infrastructure is still huge, as was painfully apparent after the earthquake and tsunami in March 2011. This disaster also underlined the inadequacy of prediction and prevention systems in current use.

One of the factors limiting full-scale implementation of these systems is the cost. There are many disaster prone areas, so in order to cover them all, a large number of systems are required. Therefore, the need for more economical disaster prediction systems has been the driving force behind numerous research projects.

As an example of one of these projects, our research group has developed an ad-hoc wireless network that can continue to operate even after a large-scale disaster [1] [3]. This is accomplished by powering the communications equipment with batteries charged by solar cells. Also, if some parts of the network are damaged, the network automatically reconfigures itself.

\section{Manuscript received July 29, 2013}

Manuscript revised February 20, 2014.

${ }^{\dagger}$ The author is with the Department of Computer Science \& Engineering, Shinshu University, Nagano-shi, 380-8553 Japan.

${ }^{\dagger}$ The author is with SCSK, Tokyo, 135-8110 Japan.

${ }^{\dagger \dagger}$ The authors are with the Integrated Intelligence Center, Shinshu University, Matsumoto-shi, 390-8621 Japan.

${ }^{\dagger+\dagger}$ The author is with Nagano Japan Radio Co. Ltd., Nagano-shi, 381-2288 Japan.

a)E-mail: david@cs.shinshu-u.ac.jp DOI: 10.1587/transcom.E97.B.1449
Our ad-hoc wireless network is currently being used in Shiojiri City, Nagano Prefecture as a regional protection system. Based on the experience gained in developing this system, we are in the process of developing a new system to predict landslides. We cannot simply transfer the network topology and protocols used in the regional protection system to make the landslide prediction system, since there are fundamental differences between the two systems that must be taken into account.

For example, in a landslide prediction sensor network, the sensor terminals are stationary and roughly equally spaced on a mountain slope. This allows us to reduce the number of transponders. However, packet collisions still occur since some terminals are hidden from one another.

To reduce packet collisions, many MAC protocols for wireless sensor networks have been proposed. In general, these protocols can be divided into TDMA-based schemes and CSMA-based schemes. CSMA-based protocols require the terminal to listen to see if another terminal is transmitting, while TDMA-based schemes require timing synchronization throughout the network.

Examples of TDMA schemes are SOTP [4], an adaptive TDMA scheme [5], FlexiTP [6] and TFDMA [7]. As with most TDMA-based schemes, an intial network setup phase to assign time slots to terminals is required.

In the LEACH protocol described in [8], TDMA is used to allocate transmission time slots for each terminal. To set up the TDMA schedule, negotiations must be carried out between the terminals and a coordinating "cluster head" before transmission can begin. This is done using CSMAbased transmission, so the system is in fact a hybrid scheme.

A similar hybrid scheme called S-MAC [9] uses periodic listen and sleep cycles to reduce power consumption, but still requires message exchanges between sensor terminals to synchronize the transmission schedule.

The Z-MAC protocol [10] is another hybrid scheme that combines the advantages of CSMA and TDMA. In this scheme, time slots have owners who are given priority to transmit. Other terminals may use the time slot if the owner has nothing to transmit. However, as with other hybrid schemes, a setup phase is required during which time slot assignments and synchronization must be done.

Our approach to transmission is similar to the Z-MAC protocol, but does not require a setup phase or synchroniza- 
tion between terminals. Instead, the terminals decide their transmission timing in a random way.

In this paper, we describe the development of our landslide prediction sensor network with particular emphasis on our MAC protocol to avoid packet loss due to collisions. Although our protocol was developed specifically for our sensor network, it can also be used in other wireless sensor networks.

\section{Landslide Detection Sensor Network}

\subsection{Overview}

An overview of our landslide detection sensor network is shown in Fig. 1 [11], [12]. Sensors buried on a mountain slope measure the water content of the soil at regular intervals. The data are collected by the sensor terminals which send the data to the server via transponders. The server processes the data to determine if a landslide is imminent.

Normally, the water content of the soil is measured every hour. When an increase in water content is detected, i.e., rainfall is absorbed by the ground, the measurement peroid is shortened in order to quickly detect possible landslide conditions. In this case, the amount of traffic through the network increases.

To test our system, we selected a mountain slope in Shiojiri City. A topological map of the area is shown in Fig. 2. According to landslide experts, sensors should be located on ridge and valley lines of the slope, so we chose the ridge lines denoted by R1-R4 and the valley lines denoted by V1$\mathrm{V} 4$ as shown in the figure.

\subsection{Network Model}

In order to determine an appropriate model to analyze our network, we positioned a sensor terminal at the locations shown by black circles in Fig. 2 in turn, then measured the received power levels at the other black circle locations. The sensor terminal transmits at a frequency of $429 \mathrm{MHz}$ with a power of $10 \mathrm{~mW}$.

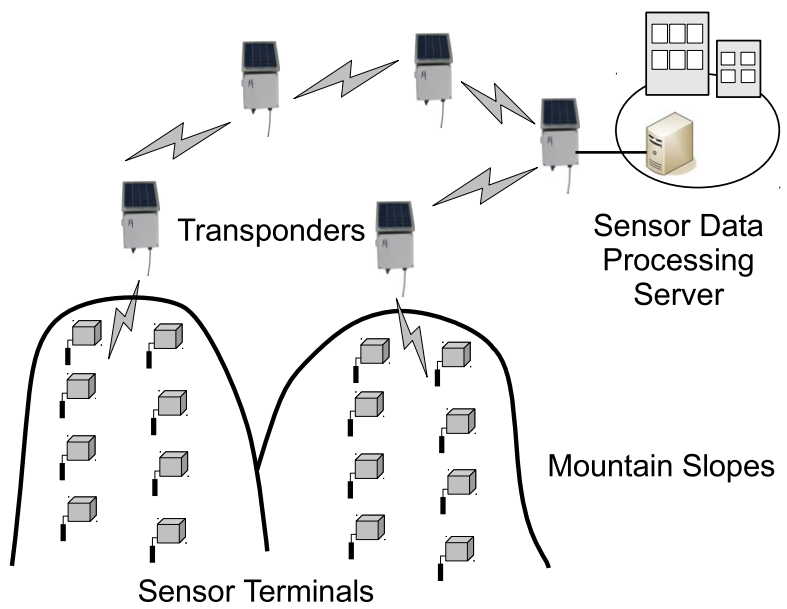

Fig. 1 An overview of our landslide prediction sensor network.
As a result of these measurements, we found that sensor terminals can sense the signal from other terminals as far away as three ridge or valley lines. For example, terminals on ridge $\mathrm{R} 3$ can sense those in valleys $\mathrm{V} 1-\mathrm{V} 4$ and ridges $\mathrm{R} 2-\mathrm{R} 4$, but cannot sense those in ridge $\mathrm{R} 1$.

Our resulting model is shown in Fig. 3. In our model, the sensor terminals are grouped along ridge or valley lines. A single transponder is used to determine the effectiveness of our collision avoidance protocol.

\subsection{Current Transmission Protocol}

In order to get our network up and running, we used the same protocol as is used in the Regional Protection System [3]. This protocol is based on the Japanese ARIB Standard STD-T67 protocol.

The basic transmission flow of the ARIB Standard protocol is shown in Fig. 4. This protocol uses carrier sensing to determine if the transmission channel is currently being

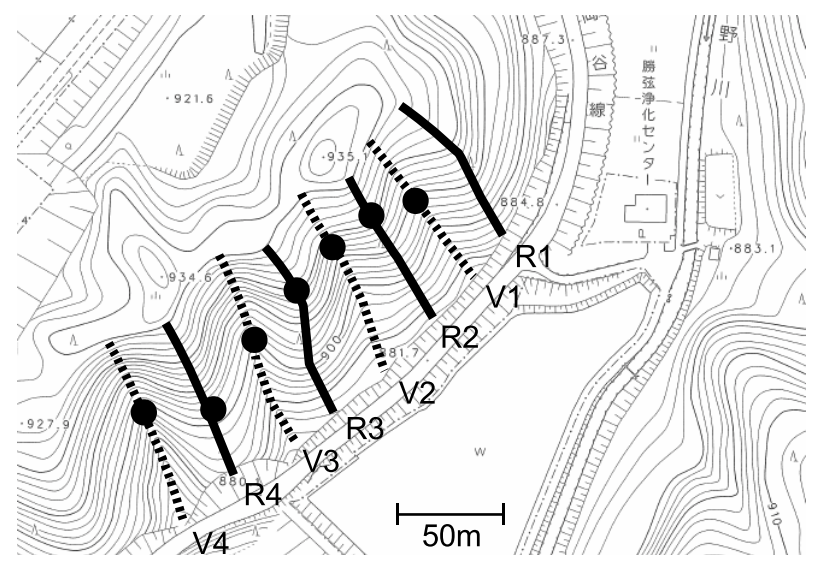

Fig. 2 A topographical map of the area where are sensor network is located. Received power levels were measured at the positions shown by black circles. R1-R4 are ridge lines of the slope and V1-V4 are valley lines.

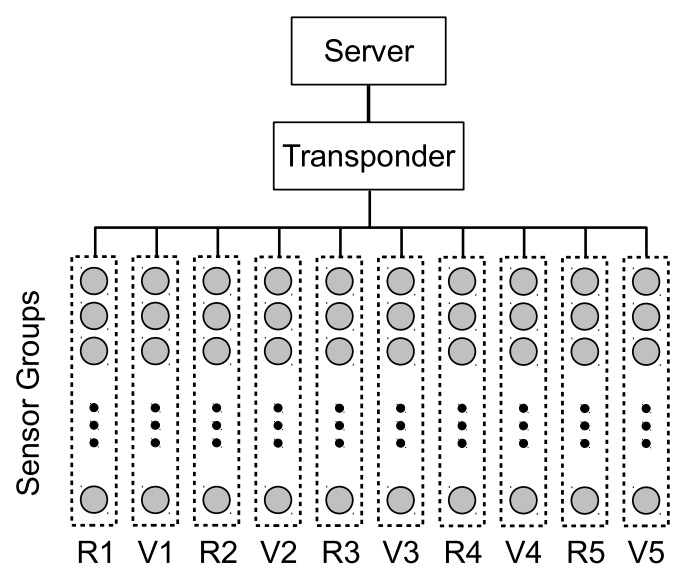

Fig. 3 A network model for our sensor network. Sensor terminals can sense other terminals as far as three groups away, e.g., sensor terminals in group R3 can sense those in V1, R2, V2, V3, R4 and V4 but not those in $\mathrm{R} 1, \mathrm{R} 5$ or V5. 


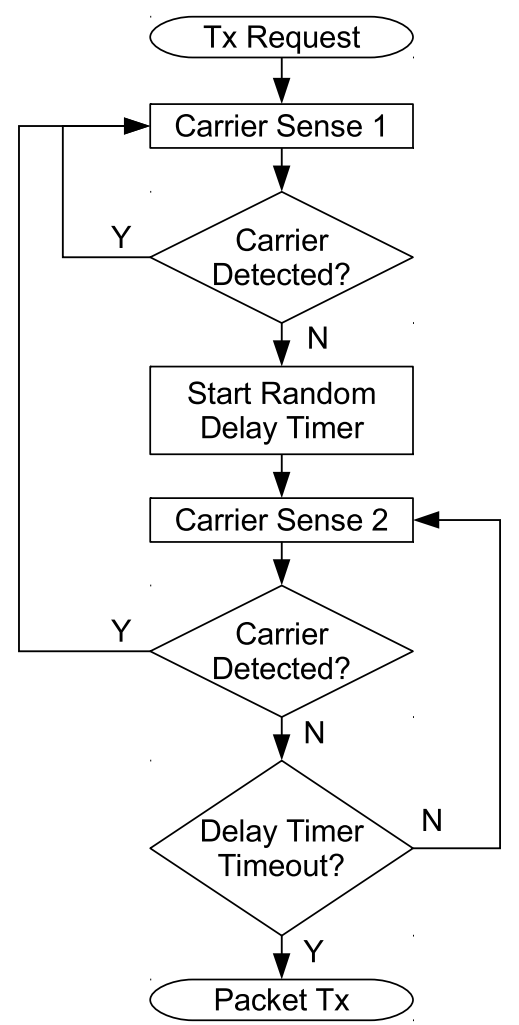

Fig. 4 The flow of the ARIB Standard STD-T67 transmission protocol. This is the current protocol used in our sensor network.

used. After the channel is found not to be in use, the channel is checked for a random amount of time before transmission.

Even though the sensor terminals use carrier sensing, collisions can still occur due to the Hidden Terminal Problem. This problem occurs in situations when two or more sensor terminals can sense the carrier of the transponder but cannot sense each other's carrier. Since the sensor terminals think that the channel is unused, they may start to transmit at approximately the same time.

To determine the limit on the number of sensor terminals that can be supported using this transmission protocol, we calculated the maximum number of sensor terminals that can transmit with zero packet loss for our network model. This limit also tells us how efficent the current protocol is.

In an ideal situation, the transmission of packets occurs as shown in Fig. 5. The sensor terminals collect data at a fixed rate of time, which determines the length of the transmission cycle. During each transmission cycle, each sensor terminal uses a time slot to send its sensor data to the server via a transponder. Since there is no space between time slots, there is no wasted transmission time.

As shown, each transmission of sensor data requires the four packet transmissions listed below before the channel is free to be used by other terminals.

- TX1: sensor data sent from terminal to transponder

- TX2: ACK sent from transponder to terminal

- TX3: sensor data sent from transponder to server

- TX4: ACK sent from server to transponder carrier sense interval

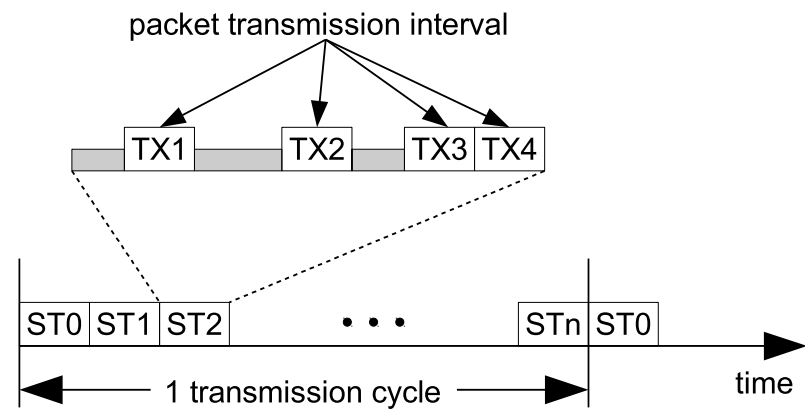

Fig. 5 Ideal transmission of packets from $n$ sensor terminals. There is no wasted transmission time.

Table 1 Carrier sense and transmission times.

\begin{tabular}{|l|c|c|}
\hline Packet Type & $\begin{array}{c}\text { Carrier Sense } \\
\text { Time (ms) }\end{array}$ & $\begin{array}{c}\text { Transmission } \\
\text { Time (ms) }\end{array}$ \\
\hline Terminal Packet (TX1) & $20-200$ & 3367 \\
\hline ACK to Terminal (TX2) & $230+(20-200)$ & 166.7 \\
\hline Transponder Packet (TX3) & $0-200$ & 3580 \\
\hline ACK to Transponder (TX4) & 0 & 206.7 \\
\hline
\end{tabular}

Table 2 Maximum number of sensor terminals with zero packet loss.

\begin{tabular}{|c|c|}
\hline Sensing Interval (minutes) & Maximum Number of Terminals \\
\hline 3 & 22 \\
\hline 10 & 73 \\
\hline 60 & 441 \\
\hline
\end{tabular}

The number of sensor terminals that can transmit during one transmission cycle depends on the time required to transmit packets. The length of the random carrier sense times and packet transmission times for the current protocol are shown in Table 1. The transmission time varies due to the length of the data packet that must be transmitted.

From Table 1, the amount of time to transmit the four packets for each sensor terminal is $7319.8 \mathrm{~ms}$. The maximum amount of time used for carrier sensing is $830 \mathrm{~ms}$, which results in a maximum tranmission time of $8149.8 \mathrm{~ms}$.

Using this value, the maximum number of sensor terminals that can ideally transmit with zero packet loss is shown in Table 2 for a few typical values of sensing interval. As shown, for sensing that is done once every 60 minutes, the maximum number of sensor terminals is 441 . As will be seen later, the current protocol can only support approximately 150 terminals when the average packet loss is about $5 \%$. Therefore, there is much room for improvement.

\section{Proposed Sensor Network Transmission Protocol}

Since power efficiency is a main design factor, we do not wish to use power for setup and timing synchronization as is required for protocols such as Z-MAC. Our proposed protocol can achieve roughly the same effect using random timing assignments, which also create temporary owners of the transmission times. This approach also makes it easier to change the number of terminals, since no setup is required. 


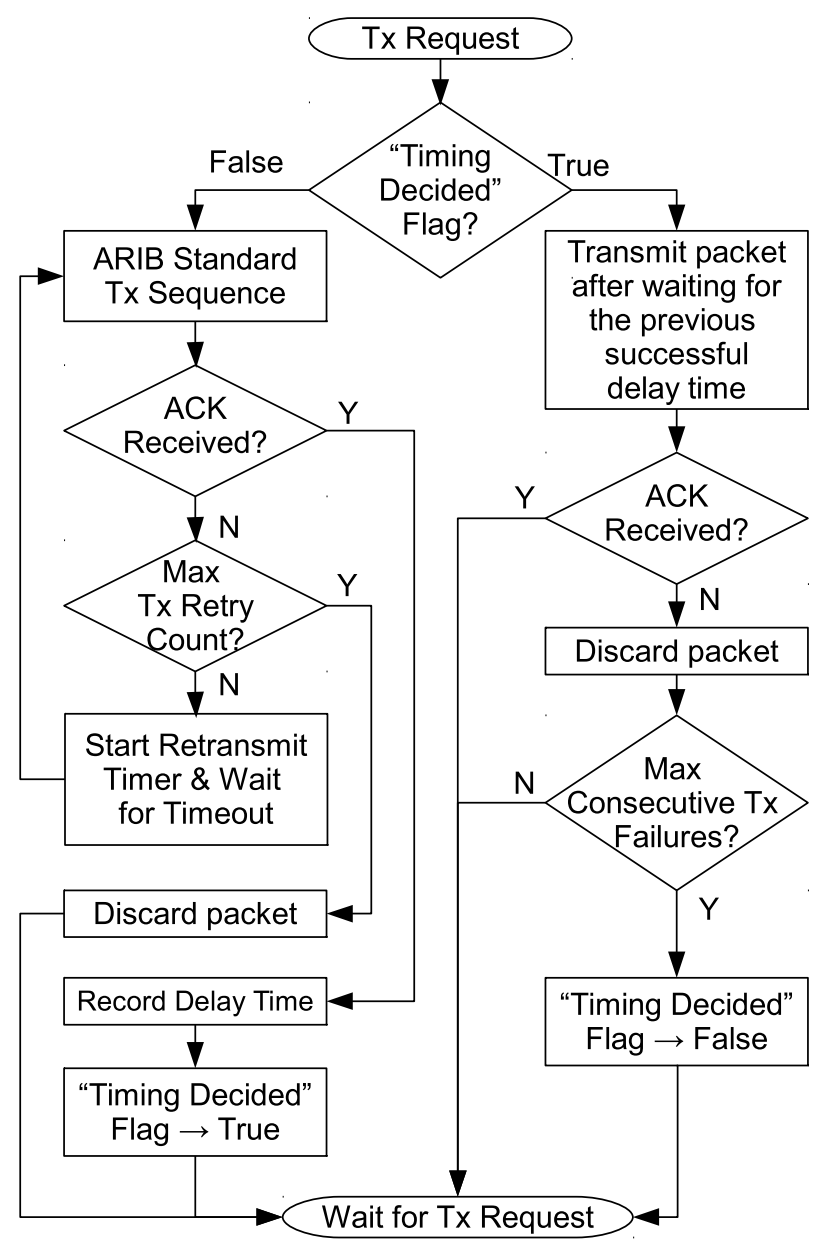

Fig. 6 Our proposed sensor network transmission protocol (Protocol 1).

Our proposed MAC protocol is outlined in Fig. 6. The basic idea is get the sensor terminals to transmit in a way similar to the situation outlined in Fig. 5. Since the terminals do not know when the other terminals will transmit, each terminal must decide the timing of their transmission by trial and error. Thus, the transmission timing is determined randomly.

When a terminal has yet to decide its timing, the "Timing Decided Flag" is false. In this case, transmission is done according to the ARIB Standard outlined in Fig. 4 with a fixed number of transmission retries. If transmission is successful, the "Timing Decided Flag" is set and the timing that was used is recorded. In subsequent transmissions, this timing is used.

Even though this timing was used successfully, it is possible that another terminal that has yet to decide its timing will use the same timing, resulting in a transmission failure. This should not happen every time, but if consecutive failures occur, the terminal should choose a different timing. In this case, the "Timing Decided Flag" is set to false.

In this way, each terminal tries different transmission times until it is able to successfully transmit. After a certain amount of time, all terminals will have their "Timing

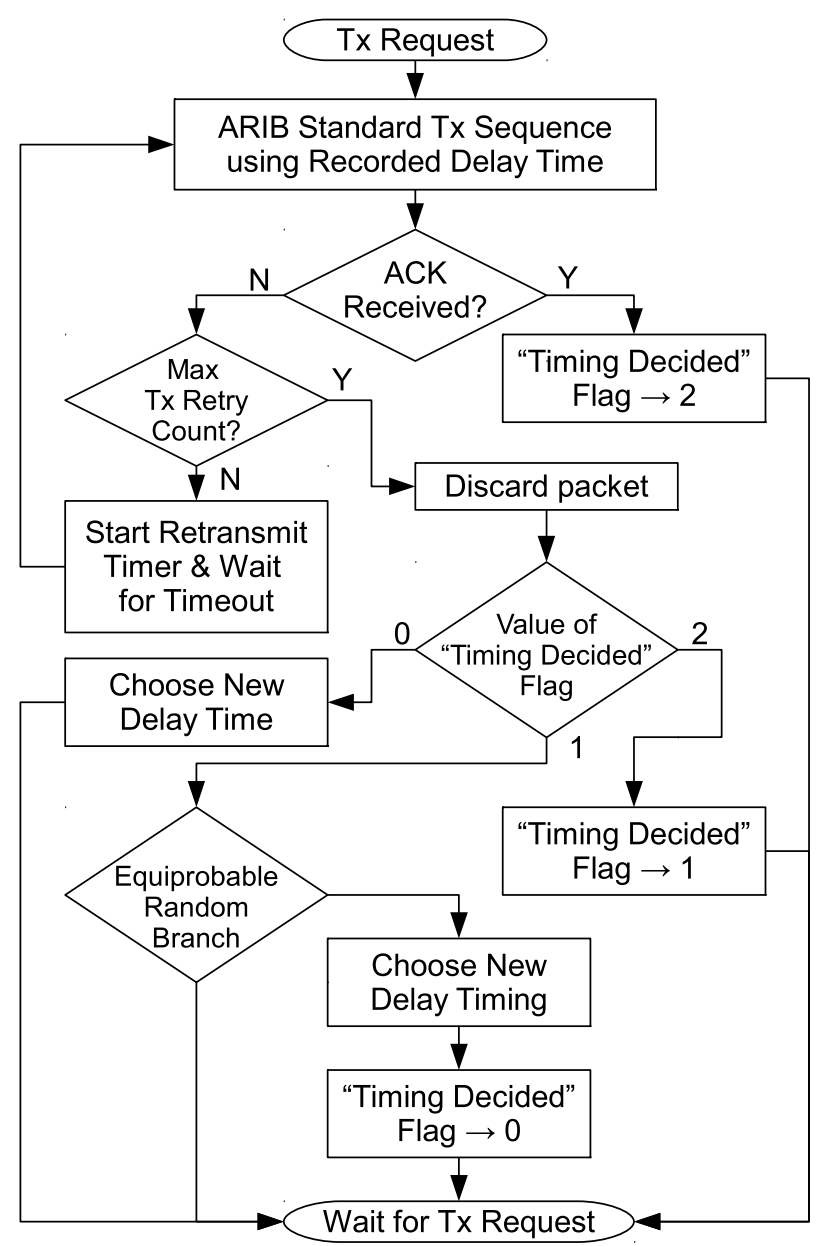

Fig. 7 Our modified transmission protocol (Protocol 2).

Decided Flag" set to true, then transmission will occur as shown in Fig. 5.

Unfortunately, there is one practical issue with this protocol. As is shown in Fig. 6, when the "Timing Decided Flag" is true, packet transmission is done without using the ARIB Standard, i.e., carrier sense is not done prior to transmission. For the radio frequency used in our system, this type of transmission is illegal in Japan.

In order to use our protocol in an actual system, we must modify it so that the ARIB Standard transmission sequence is used for every transmission. Taking this into consideration, we modified our transmission protocol as shown in Fig. 7.

As shown, transmission begins with the ARIB Standard sequence with a fixed number of transmission retries. If the transmission is successful, i.e., an ACK is received, the "Timing Decided Flag" is set to 2. If the transmission fails, the packet transmission sequence is retried until the maximum number of retries has been reached. At this point, since the packet could not be sent, the packet is discarded and the transmission timing used next time is selected depending on the current value of the "Timing Decided Flag". For terminals that have successfully transmitted, i.e., their "Timing 
Decided Flag" is equal to 2, the same timing is used, but the value of the "Timing Decided Flag" is set to 1 . For terminals that have their "Timing Decided Flag" equal to 1, a new random timing is used with a probability of $1 / 2$. Terminals that have not decided their timing, i.e., their "Timing Decided Flag" is set to 0 always use a new random timing.

\section{Performance Evaluation}

To evaluate our proposed protocols, we used computer simulations using the parameters listed in Table 3. The carrier sense and transmission times are the same as those shown in Table 1. Evaluations were done for sensing intervals of 10 minutes and 60 minutes. However, since the trends for both were the same, we mainly show results for a sensing interval of 60 minutes.

The measure of performance used was the average packet loss probability. If the number of packets created by the sensor terminals is $N_{t}$ and the number of packets received by the server is $N_{s}$, we define the average packet loss probability, $\bar{P}_{l}$, as

$$
\bar{P}_{l}=100\left(1-\frac{N_{s}}{N_{t}}\right) .
$$

When the number of transmission retries is equal to 7 , the average packet loss probability of the current protocol and our two proposed protocols is shown in Fig. 8 for a sensing interval of 60 minutes and in Fig. 9 for a sensing interval of 10 minutes. For reference, the theoretical maximum number of terminals possible with $0 \%$ packet loss, 441 for a 60 minute sensing interval and 73 for a 10 minute sensing interval, is shown as a vertical line.

We can see that Protocol 1 is a vast improvement over the current protocol. For example, for an average packet loss probability of approximately 10\%, the current protocol can only support about 180 terminals, but Protocol 1 can support about 290 terminals, while Protocol 2 can support about 250 terminals when the sensing interval is 60 minutes. For a sensing interval of 10 minutes, the corresponding numbers of terminals are 32, 55 and 42 respectively. Protocol 2 is a realistic compromise to Protocol 1 , so its performance is degraded, but is still better than the current protocol.

Table 3 Simulation parameters.

\begin{tabular}{|c|c|}
\hline Parameter & Value \\
\hline Bit Rate & $1200 \mathrm{bps}$ \\
\hline Sensing Interval & $10 \mathrm{~min} ., 60 \mathrm{~min}$. \\
\hline Simulation Time & $30,000 \mathrm{~min}$. \\
\hline Simulation Trials & minimum of 10 \\
\hline Tx. Retries & 2,7 \\
\hline Random delay for retry timer & $0-10 \mathrm{~s}(2 \mathrm{~s}$ intervals $)$ \\
\hline $\begin{array}{c}\text { Max. consecutive Tx. failures before } \\
\text { a new timing is tried (Protocol 1) }\end{array}$ & $0-3560 \mathrm{~s}(40 \mathrm{~s}$ intervals) \\
\hline $\begin{array}{c}\text { New random timing } \\
\text { (Protocol 2, 60 min. sensing interval) }\end{array}$ & $0-593.3 \mathrm{~s}(6.7 \mathrm{~s}$ intervals) \\
\hline $\begin{array}{c}\text { New random timing } \\
\text { (Protocol 2, 10 min. sensing interval) }\end{array}$ & 0 \\
\hline
\end{tabular}

Looked at from a different perspective, when 250 sensor terminals are used with a sensing interval of 60 minutes, the average packet loss probability is only about $4 \%$ for Protocol 1, but is about $17 \%$ for Protocol 2 and $76 \%$ for the current protocol. Therefore, the number of sensor terminals that can be supported is vastly improved using our proposed protocols.

Since the maximum number of terminals that can be supported is smaller when the sensing interval is shorter, the avereage packet loss probability is larger for a sensing interval of 10 minutes. However, we can see that the trends for the three protocols shown are the same. Therefore, in the following results we focus on a sensing interval of 60 minutes.

When the number of retries is reduced to 2 , the packet loss probability increases more slowly as the number of sensor terminals increases as shown in Fig. 10. In this case, Protocol 2 has a lower average packet loss probability when the number of sensor terminals is less than about 280 , but

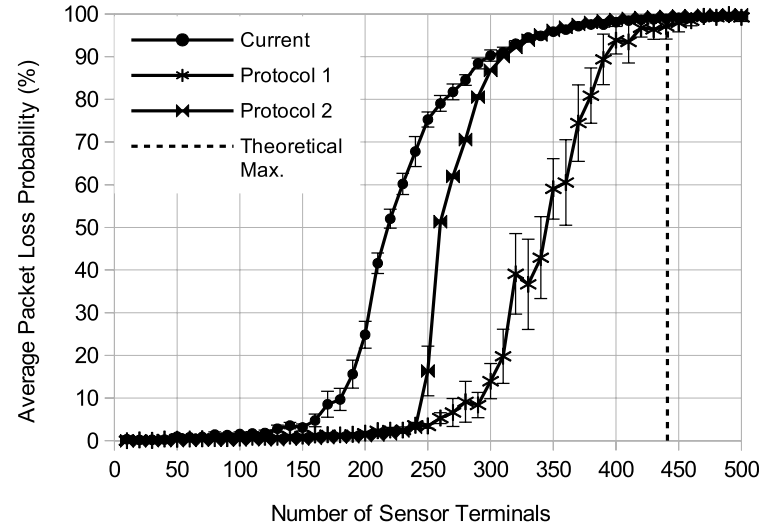

Fig. 8 The average packet loss probability as a function of the number of sensor terminals for the current protocol and the two proposed protocols when the number of retries is 7 and the sensing interval is 60 minutes. The theoretical maximum number of terminals is 441 . The error bars show the $95 \%$ confidence interval.

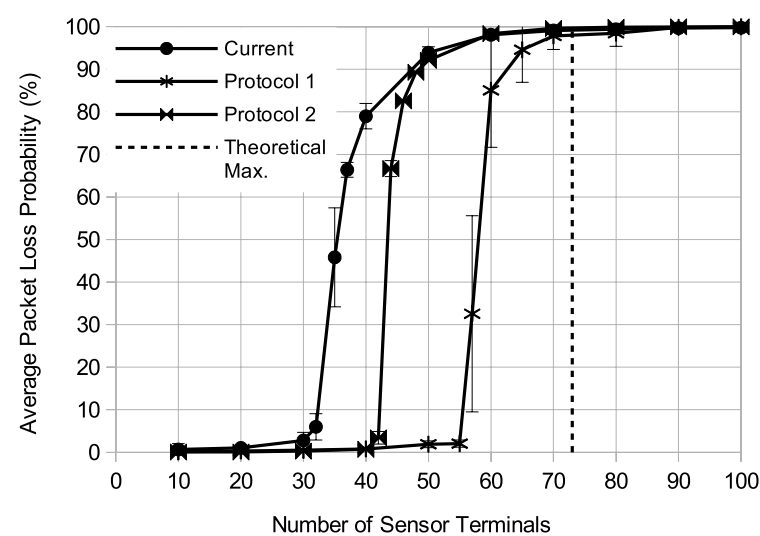

Fig. 9 The average packet loss probability as a function of the number of sensor terminals for the current protocol and the two proposed protocols when the number of retries is 7 and the sensing interval is 10 minutes. The theoretical maximum number of terminals is 73 . The error bars show the $95 \%$ confidence interval. 


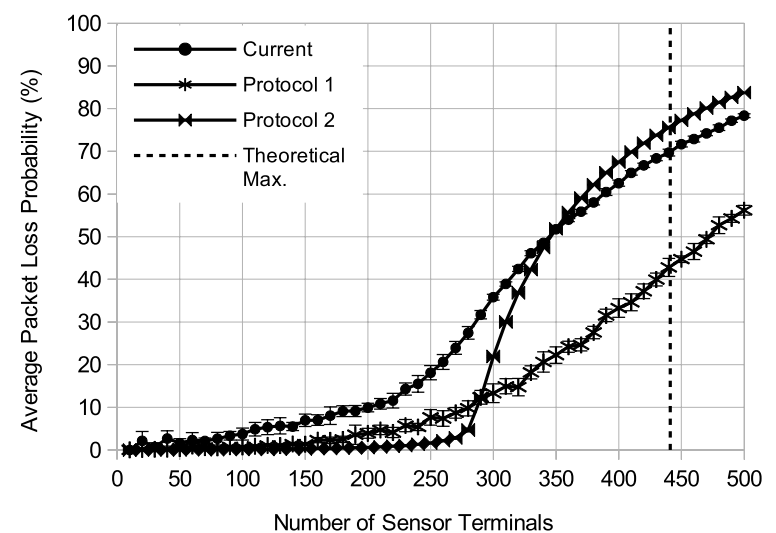

Fig. 10 The average packet loss probability as a function of the number of sensor terminals for the current protocol and the two proposed protocols when the number of retries is 2 and the sensing interval is 60 minutes. The theoretical maximum number of terminals is 441 . The error bars show the $95 \%$ confidence interval.

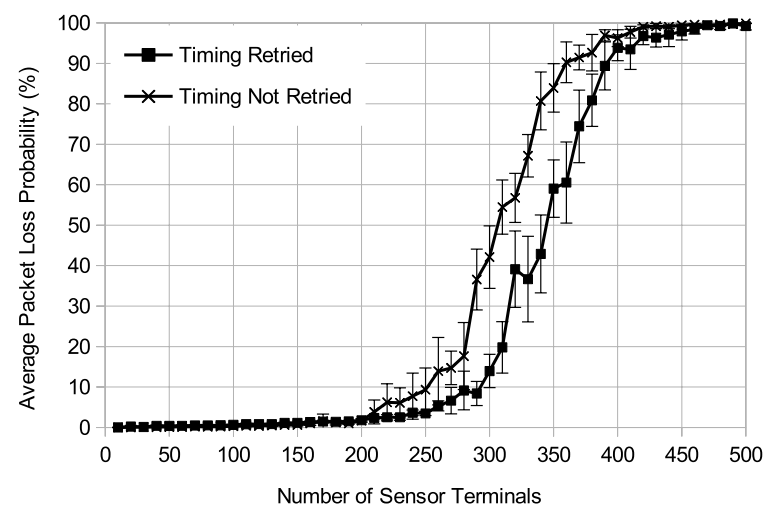

Fig. 11 The effect of retrying the same transmission timing after a transmission failure for Protocol 1. "Timing Not Retried" is equivalent to setting the "Max. consecutive Tx. failures before a new timing is tried" parameter equal to 0 . The sensing interval is 60 minutes. The error bars show the $95 \%$ confidence interval.

rapidly worsens when more terminals are added. On the other hand, Protocol 1 still has better performance than the current protocol.

Comparing the results in Fig. 8 and Fig. 10, when the number of retries is reduced, the overall traffic in terms of number of packets is reduced, which results in an overall reduction in packet loss. However, when the number of terminals is small, the packet loss probability increases because of the lower number of retries.

To evaluate the effect of using the same timing after a transmission failure, we determined the average packet loss probability when the same timing was not retried. The results are shown in Fig. 11 for Protocol 1 and Fig. 12 for Protocol 2 compared to the performance when the timing is retried.

As the results show, retrying the same timing improves the performance for both protocols. This shows the effectiveness of our approach, which aims to have each terminal determine its timing randomly. A few transmission failures

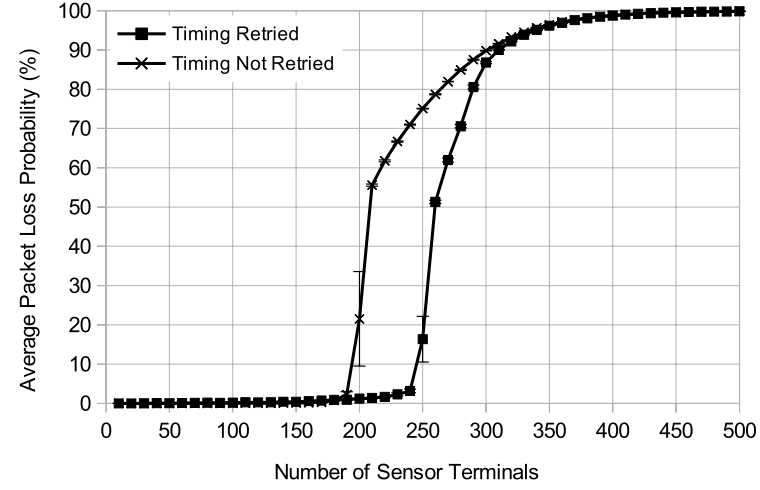

Fig. 12 The effect of retrying the same transmission timing after a transmission failure for Protocol 2. "Timing Not Retried" is equivalent to setting the "Timing Decided Flag" to 0 instead of 1, i.e., the "Timing Decided Flag" $=1$ branch of the algorithm is not used. The sensing interval is 60 minutes. The error bars show the $95 \%$ confidence interval.

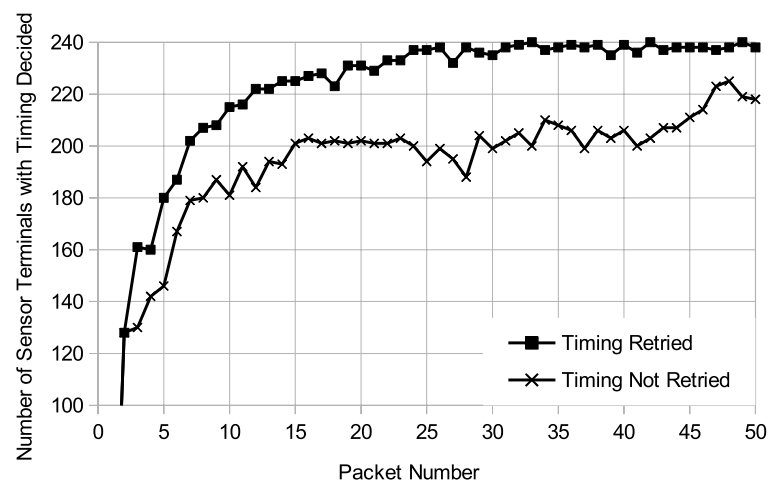

Fig. 13 The number of terminals that have decided their transmission timing using Protocol 1 as a function of the number of packets transmitted when the same timing is retried and when it is not retried. The total number of terminals is 240 . The sensing interval is 60 minutes. The error bars show the $95 \%$ confidence interval.

can result from terminals that have not decided their timing yet, so it is important for terminals that have decided their timing to retry the same timing.

Another way to show the effectiveness of retrying the same transmission timing is to look at the number of terminals that have decided their timing. When 240 sensor terminals are used, the number of terminals that have decided their timing as a function of the number of packets transmitted is shown in Fig. 13 for Protocol 1 and Fig. 14 for Protocol 2.

For Protocol 1, we can see that almost all of the terminals can decide their timing after transmitting only about 25 packets when the same timing is retried. However, when the same timing is not retried, it is more difficult for all the terminals to decide their timing.

This difference is more pronounced for Protocol 2. Even after 50 packets are transmitted, only a little more than half of the terminals have decided their timing when the same timing is not retried. 


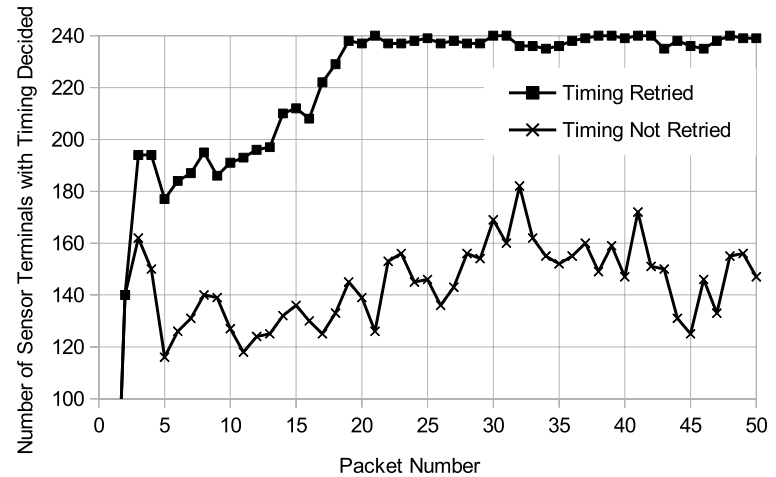

Fig. 14 The number of terminals that have decided their transmission timing as a function of the number of packets transmitted when the same timing is retried and when it is not retried for (algorithm 2). The total number of terminals is 240 . The sensing interval is 60 minutes. The error bars show the $95 \%$ confidence interval.

\section{Conclusions}

In this paper, we introduced our landslide prediction sensor network currently being tested in Shiojiri, Japan. For this network, we proposed a new MAC protocol that vastly reduces the average packet loss probability, which in turn allows more sensor terminals to be used.

The basic idea of our protocol is to have the sensor terminals decide their transmission timing in a random way, so that the overall transmission of packets is close to ideal, i.e., transmission time is not wasted. Our protocol also has the advantage that timing setup and synchronization throughout the network are not necessary. Therefore, a timing beacon does not need to be transmitted, which leads to low power consumption.

Simulation results showed that the number of sensor terminals can be increased from about 180 terminals to more than 250 terminals at an average packet loss probability of $10 \%$. For a fixed number of sensor terminals, the proposed protocol reduced the average packet loss probability. For example, when there are 250 terminals, the probability was reduced from $76 \%$ to less than $17 \%$.

Our protocol was designed for our landslide prediction sensor network, but it can also be used in other wireless sensor networks.

\section{References}

[1] Y. Fuwa, "High fault resilient adhoc network systems," J. IEICE, vol.91, no.10, pp.862-864, Oct. 2008 (in Japanese).

[2] K. Nakanishi, S. Horio, M. Niimura, H. Kunimune, E. Motoyama, and Y. Fuwa, "Development and evaluation for regional protection system using a wireless ad-hoc network," IEICE Technical Report, CS2008-27, 2008 (in Japanese).

[3] Y. Fuwa, S. Horio, K. Nakanishi, M. Niimura, H. Kunimune, and E. Motoyama, "Regional protection system using a wireless ad-hoc network," IEICE Technical Report, AN2008-38, 2008 (in Japanese).

[4] Y. Wang, I. Henning. X. Li, and D. Hunter, "SOTP: A selforganized TDMA protocol for wireless sensor networks," Proc. IEEE CCECE/CCGEI, pp.1108-1111, May 2006.
[5] T.-H. Hsu and P.-Y. Yen, "Adaptive time division multiple accessbased medium access control protocol for energy conserving and data transmission in wireless sensor networks," IET Commun. vol.5, no.18, pp.2662-2672, 2011.

[6] W.L. Lee, A. Datta, and R. Cardell-Oliver, "FlexiTP: A flexibleschedule-based TDMA protocol for fault-tolerant and energyefficient wireless sensor networks," IEEE Trans. Parallel Distrib. Syst., vol.19, pp.851-864, June 2008.

[7] D. Buranapanichkit and Y. Andreopoulos, "Distributed timefrequency division multiple access protocol for wireless sensor networks," IEEE Wireless Commun. Lett., vol.1, pp.440-443, Oct. 2012.

[8] W.B. Heinzelman, A.P. Chandrakasan, and H. Balakrishnan, "An application-specific protocol architecture for wireless microsensor networks," IEEE Trans. Wireless Commun., vol.1, pp.660-670, Oct. 2002.

[9] W. Ye, J. Heidemann, and D. Estrin, "Medium access control with coordinated adaptive sleeping for wireless sensor networks," IEEE/ACM Trans. Netw., vol.12, pp.493-506, June 2004.

[10] I. Rhee, A. Warrier, M. Aia, J. Min, and M.L. Sichitiu, "Z-MAC: A hybrid MAC for wireless sensor networks," IEEE/ACM Trans. Netw., vol.16, pp.511-524, June 2008.

[11] D. Kuroyanagi, D.K. Asano, H. Suzuki, and Y. Fuwa, "A sensor network terminal for disaster detection," IEICE Technical Report, ICSSSL2011-05, 2011 (in Japanese).

[12] H. Suzuki, D. Kuroyanagi, D.K. Asano, M. Komatsu, Y. Takeshita, K. Sawada, M. Futagawa, and Y. Fuwa, "Construction of a sensor network to forecast landslide disasters using an ad-hoc network and EC sensors," IEICE Technical Report, ICSSSL2012-1, 2012 (in Japanese).

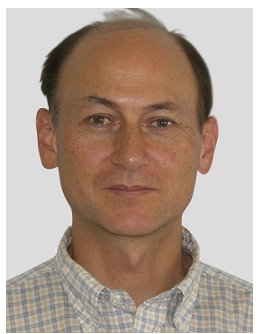

David Asano received the B.A.Sc. degree (with honours) from the University of British Columbia, Canada, and the M.A.Sc. and Ph.D. degrees from the University of Toronto, Canada, all in Electrical Engineering. He was an STA Fellow at the Communications Research Laboratory of the Ministry of Posts and Telecommunications in Tokyo, Japan from 1994 until 1996. In 1996, he joined the Department of Computer Science \& Engineering, Faculty of Engineering, Shinshu University, Nagano, Japan, where he is now an Associate Professor. Since 2001, he has also been a part-time lecturer at the Nagano National College of Technology. His current research interests include coding and modulation techniques, sensor networks and information systems. Dr. Asano is a Senior Member of the IEEE and IEICE.

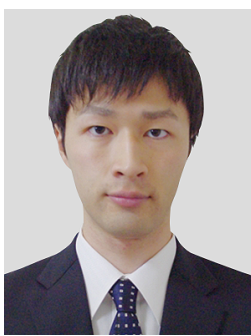

Daichi Kuroyanagi received the M.E. degree in Computer Science \& Engineering from Shinshu University. He is currently working for SCSK Corporation in Tokyo Japan. 


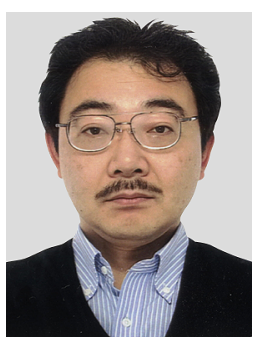

Hikofumi Suzuki received the B.E. and M.E. degrees from Shinshu University, Nagano, Japan in 1992 and 1994 respectively. From 1997 to 2003 , he was a research associate in the Department of Electronics and Computer Science, Nagano National College of Technology, Japan. From 2003 to 2005, he was a research associate in the Graduate School of Frontier Sciences, University of Tokyo, Japan. From 2005 to 2009, he was an Associate Professor in the General Information Processing Center, Shinshu University. Since 2009, he has been an Associate Professor in and the Vice Director of the Integrated Intelligence Center, Shinshu University. His current research areas include Networks, Distributed Systems and Sensor/AdHoc Networks. He is a member of JSiSE, IEICE and IPSJ.

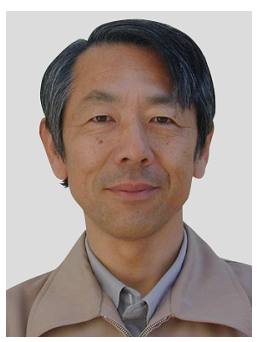

Eiki Motoyama graduated from Tokyo University of Science, Department of Electrical Engineering in 1979 and in the same year, joined Japan Radio Co., Ltd. In JRC, he was involved in the development of wireless equipment. In 1986, he joined Nagano Japan Radio Co., Ltd. and became involved in the development of communication equipment and communication systems. From 2005, he became involved in research and development of wireless networks. He is currently manager of the Communication Engineering Department, Engineering Group 2.

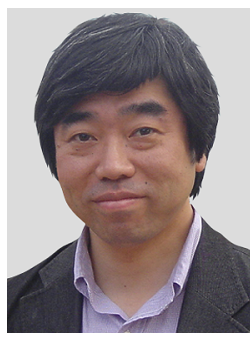

Yasushi Fuwa received his B.E. and M.E degrees from Shinshu University in 1981 and 1983, respectively. From 1983 to 1992, he was a research associate in the Faculty of Engineering, Shinshu University. He received his Ph.D. from Nagoya Institute of Technology in 1992. $\mathrm{He}$ is now a professor in the Graduate School of Science and Technology, Shinshu University. His current research areas include Ad-Hoc networks, sensor networks, computer networks and e-Learning. He is a member of JSiSE, IEICE,

IPSJ and IEEE. 